\title{
Modelación hidrodinámica y determinación de la calidad del agua en el río Botello, Facatativá, Cundinamarca, Colombia
}

\section{Hydrodynamic modeling and determination of the water quality in the river Botello, Facatativá, Cundinamarca, Colombia}

\section{Modelagem hidrodinâmico e determinação da qualidade da água em rio Botello, Facatativá, Cundinamarca, Colômbia}

\author{
Angel Oswaldo Antonio Paiba' \& Edinson Fabian Monroy Avila ${ }^{2}$ \\ 1,2Estudiante de Ingeniería Ambiental \\ 1,2 Grupo de Investigación Axioma. Facultad de Ciencias agropecuarias e ingeniería. \\ Universidad de Cundinamarca. Facatativá, Cundinamarca.
}

swald026@gmail.com¹, ingenierofabianm@gmail.com²

\section{Resumen}

La presente investigación plantea estudiar el comportamiento hidrodinámico de un tramo del Río Botello comprendido en la zona de influencia de la cabecera municipal de Facatativá, departamento de Cundinamarca, Colombia. En la primera fase, se determinaron las variables hidrodinámicas esenciales para realizar el modelamiento hidrodinámico, además, se determinaron las variables para estudiar la calidad del agua. Para la parte hidrodinámica, se recopiló información de velocidades, áreas transversales, y batimetría. Con respecto a la calidad, se determinaron temperatura, acidez, color, coliformes, sólidos disueltos, DBO5, DQO, nitratos, nitritos, manganeso y hierro. En la segunda fase, se procesó la información para finalmente generar el modelamiento hidrodinámico del río Botello. Se concluyó que la modelación del río Botello se constituye como una herramienta indispensable para la gestión integral del recurso hídrico en el municipio de Facatativá . En los puntos de muestreo hay razones de variación de las variables hidrodinámicas de acuerdo con el tramo analizado y de la calidad del agua, las cuales están fuertemente influenciadas por las actividades agropecuarias e industriales que se realizan en la zona circundante del sistema hídrico, lo cual acarrea grandes problemas de calidad a la oferta del recurso hídrico para los habitantes del municipio que suplen sus necesidades básicas del río Botello. En cuanto a inundaciones, se precisa que el municipio de Facatativá se encuentra en una zona de riesgo en períodos de alta precipitación.

Palabras clave: calidad de agua, evaluación de riesgo, inundación, modelación hidrodinámica

\section{Abstract}

This research proposes to study the hydrodynamic behaviour of a section of the Botello River within the area of influence of the municipality of Facatativa, department of Cundinamarca, Colombia. In the first phase, essential hydrodynamic variables were determined for the hydrodynamic modeling, additionally, variables to study the quality of the 
water were determined. For the hydrodynamic phase, data of speed, cross-cutting areas, and bathymetry were collected. With respect to the quality, data for temperature, acidity, colour, coliforms, dissolved solids, BOD5, cod, nitrates, nitrites, manganese and iron were determined. In the second phase, the information was processed to finally generate the hydrodynamic modeling of river Botello. It was concluded that modeling of river Botello is an indispensable tool for the integrated management of water resources in the municipality of Facatativa. There are reasons for variation of the hydrodynamic variables according to the analyzed section and the water quality, which are strongly influenced by agricultural and industrial activities carried out in the surrounding area of the water system, which could bring great problems of quality in the supply of the water resource to the inhabitants of the municipality those who satisfy their basic needs of river Botello in sampling points. In terms of flooding, it was establish that the municipality of Facatativa is a risk zone during periods of high rainfall.

Key-words: water quality, assessment of risk, flood, hydrodynamic modeling

\section{Resumo}

A presente pesquisa tem como propósito estudar o comportamento hidrodinâmico de um trecho do rio Botello que se encontra na zona de influencia do município de Facatativá, estado de Cundinamarca, Colômbia. Na primeira fase, se determinou as variáveis hidrodinâmicas essenciais para realizar a modelagem, além de determinar as variáveis úteis para estudar a qualidade da água. Para o estudo hidrodinâmico, se registrou informação de velocidades, áreas transversais e batimetría. A respeito da qualidade, se determinou temperatura, acidez, cor, coliformes, sólidos dissolvidos, DB05, DQO, nitratos, nitritos, manganês e ferro. Na segunda fase foi processada a informação para finalmente gerar a modelagem hidrodinâmica do rio Botello. Foi concluído que a modelagem do rio Botello constitui uma ferramenta indispensável para a gestão integral do recurso hídrico no município de Facatativá. Nos pontos de amostragem, há razões de variação das variáveis hidrodinâmicas de acordo com o tramo analisado e da qualidade da água, as quais estão fortemente influenciadas pelas atividades agropecuárias e industriais que se realizam na zona circundante do sistema hídrico para os moradores do município que satisfazem suas necessidades básicas do rio Botello. Quanto a inundações, sería necessário que o município de Facatativá estivesse em uma zona de risco em períodos de alta precipitação.

Palavras-chave: qualidade da água, avaliação de risco, inundação, modelagem hidrodinâmica

\section{Introducción}

Las tradicionales causas de deterioro del recurso hídrico, son la deforestación especialmente en áreas altas de la cuenca, el deseo de expandir la frontera agrícola para cultivos tradicionales de la región, la urbanización causada por fenómenos culturales, el aumento de vertimientos sin control o tratamiento, y el aumento de residuos sólidos, entre otros, (Mautaco, 2014)

La cabecera municipal de Facatativá, se abastece de agua del Río Botello y fuentes alternativas, tales como el "Río Subachoque y quebradas afluentes 
como: Mancilla, San Rafael y La Pava". (Alcaldia municipal de Facatativá, 2001), que suplen la demanda hídrica en temporada seca o períodos de bajos niveles de precipitación, para tener "cobertura de servicio de acueducto de aproximadamente un 94,63\%" (Ministerio de vivienda, 2010).

En esta investigación, es utilizado el modelo matemático unidimensional Hec-Ras 4.1.0., software gratuito de origen Estadounidense desarrollado por parte del Cuerpo de Ingenieros de la Armada (US Army Corps of Engineering). La investigación propuesta se orientó a modelar hidrodinámicamente y evaluar la calidad del agua del Río Botello, sistema hídrico de gran importancia para el municipio de Facatativá, Cundinamarca, Colombia. (Secretaria de Desarrollo Agropecuario, 2012)

Este trabajo, sienta un precedente importante en el ámbito social, ambiental, económico y académico del sector. El modelamiento de procesos ambientales se aproxima a una realidad del fenómeno, que lo hace predecible, lo cual, brinda beneficios de prevención y mitigación de desastres naturales.

De esta manera, la obtención de parámetros hidráulicos, permite conocer la posible ocurrencia de eventos hidrometeorológicos que pueden impactar catastróficamente al municipio, centrando la necesidad en la predicción del riesgo, asociada a la amenaza hidrológica (nivel del agua) más que en la atención del desastre.

\section{Materiales y métodos}

El Río Botello, nace en el Cerro Peñas del Aserradero en la Vereda La Tribuna, a una altura superior de $3.000 \mathrm{msnm}$, tiene una extensión de $189 \mathrm{~km}^{2}$ localizados en jurisdicción de los municipios de Facatativá, Bojaca, Madrid y Mosquera lo cual según la guía básica para la caracterización morfométrica de cuencas hidrográficas establecida por la Universidad del Valle (2010) la clasifica como sub-cuenca.

La red de drenaje es poco densa, dadas las características del suelo, el subsuelo y material parental; presenta una dirección predominante NWSE, y en su recorrido de aproximadamente 20 kilómetros de longitud, 5,3 kilómetros son la zona de influencia en la cabecera municipal, lugar de estudio, tomando como inicio el cruce del rio con la calle 1 vía Villeta - Bogotá en las coordenadas $4^{\circ} 49.339 ',-74^{\circ} 22.324^{\prime}$ pasando por la cabecera del municipio y terminando en las coordenadas $4^{\circ}$ 48.233', $-74^{\circ} 20.370$

La primera fase de la investigación consistió en el levantamiento y recopilación de información primaria, a través de muestreos, en los puntos establecidos en la Tabla 1 y Figura 1 . En estos puntos se determinan las variables: velocidad superficial del agua, ancho de la superficie libre, profundidades, levantamiento de los perfiles, que fueron necesarias para el modelamiento hidrodinámico.

Tabla 1. Coordenadas de los puntos de muestreo.

\begin{tabular}{lcc}
\hline $\begin{array}{l}\text { Punto de } \\
\text { muestreo }\end{array}$ & $\begin{array}{c}\text { Coordenadas } \\
\text { E }\end{array}$ & $\begin{array}{c}\text { Coordenadas } \\
\text { N }\end{array}$ \\
\hline punto 1 & 967323 & 1025034 \\
punto 2 & 967606 & 1024699 \\
\hline punto 3 & 968341 & 1024034 \\
\hline punto 4 & 968417 & 1023800 \\
\hline punto 5 & 968549 & 1023686 \\
\hline punto 6 & 968690 & 1023465 \\
\hline punto 7 & 968879 & 1023223 \\
\hline punto 8 & 969022 & 1023006 \\
\hline punto 9 & 969874 & 1022958 \\
\hline
\end{tabular}




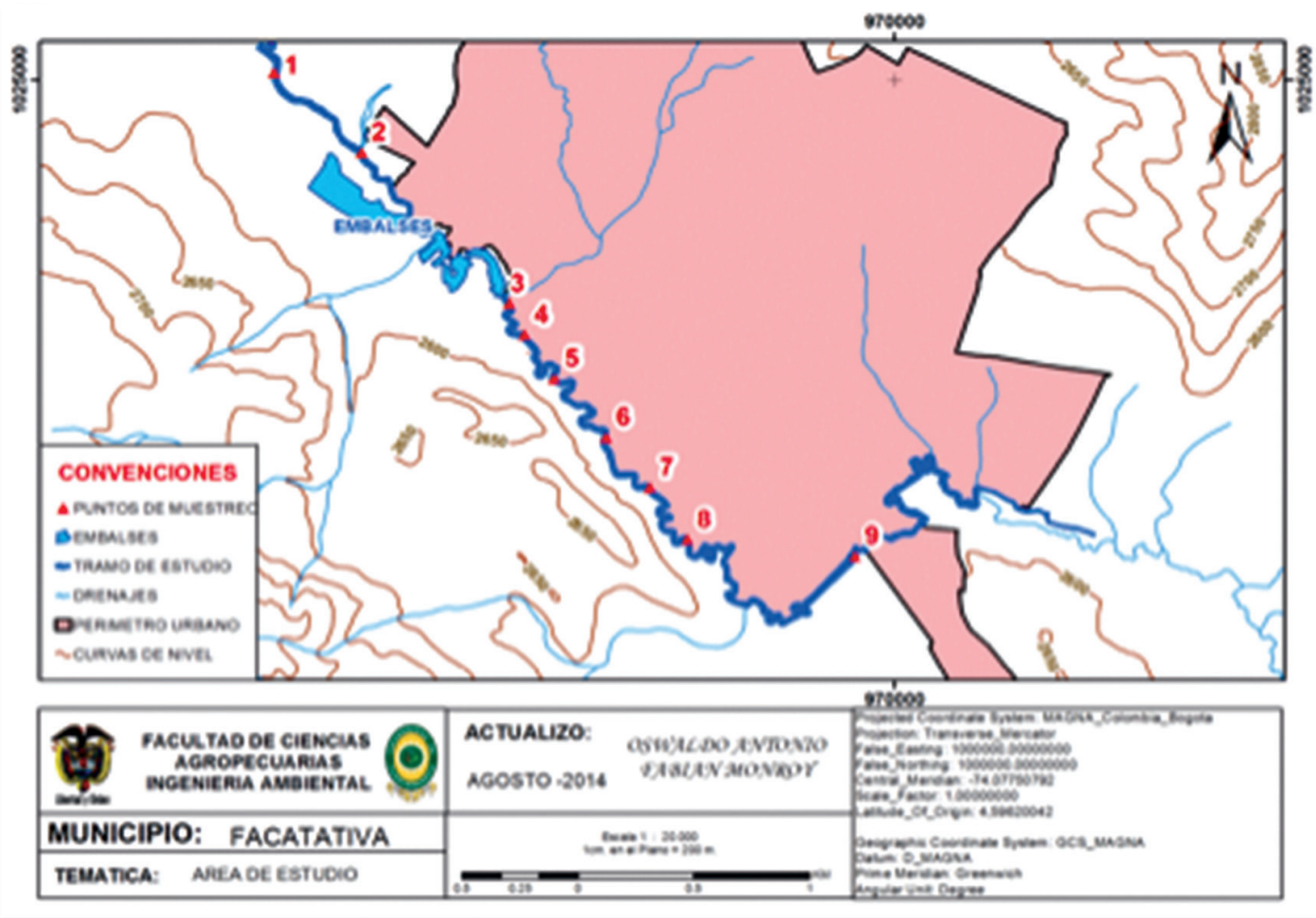

Figura 1. Ubicación de los puntos de muestreo.

Se realizaron tres muestreos sistemáticos y en cada uno se tomaron siete muestras de agua, como lo indican los puntos de muestreo (Figura 2). Las muestras se tomaron de acuerdo a la metodología establecida por las Normas Técnicas Colombiana (NTC) 813 y (NTC) 5667-6, incluidas en las normas oficiales para la calidad del agua. (Icontec, 1996)

La determinación de los parámetros de calidad se llevó a cabo mediante el muestreo in-situ y el posterior análisis de las mismas, en los laboratorios de la empresa Aguas de Facatativá, en las cuales se determinaron $\mathrm{PH}$, temperatura, cloruros, color aparente, conductividad, DBO5, DQO, fosfatos, hierro total, nitratos, nitritos, manganeso, oxígeno disuelto, sólidos disueltos totales, turbiedad, y coliformes totales

Además, se empleó el método de filtración por membranas para los análisis microbiológicos, las variables fisicoquímicas, y se realizaron análisis fotométricos, volumétricos y electrométricos respectivamente.
Posteriormente se realizó el procesamiento de la información obtenida, la calibración del software, y el modelamiento de los escenarios hidrodinámicos en el software HEC-Ras (Us army corps of engineers, 2010). Según la metodología planteada por Blade (2009) Los pasos básicos para desarrollar un modelo con HEC-RAS, se pueden resumir en 5 puntos: Iniciar un nuevo proyecto, ingresar los datos de geometría y de las secciones, introducir los caudales y condiciones de contorno, ejecutar los cálculos hidráulicos., visionar, calibrar y validar los resultados.

Por otro lado, el método empleado para determinar la calidad del agua fue definido con base en la metodología establecida en el Standard Methods for Examination of Water and Wastewater (American Public Health Association, 2012). Finalmente, los resultados obtenidos se analizaron fundamentándose en parámetros establecidos por la guía Water Quality Assessments (Who, 1996) 


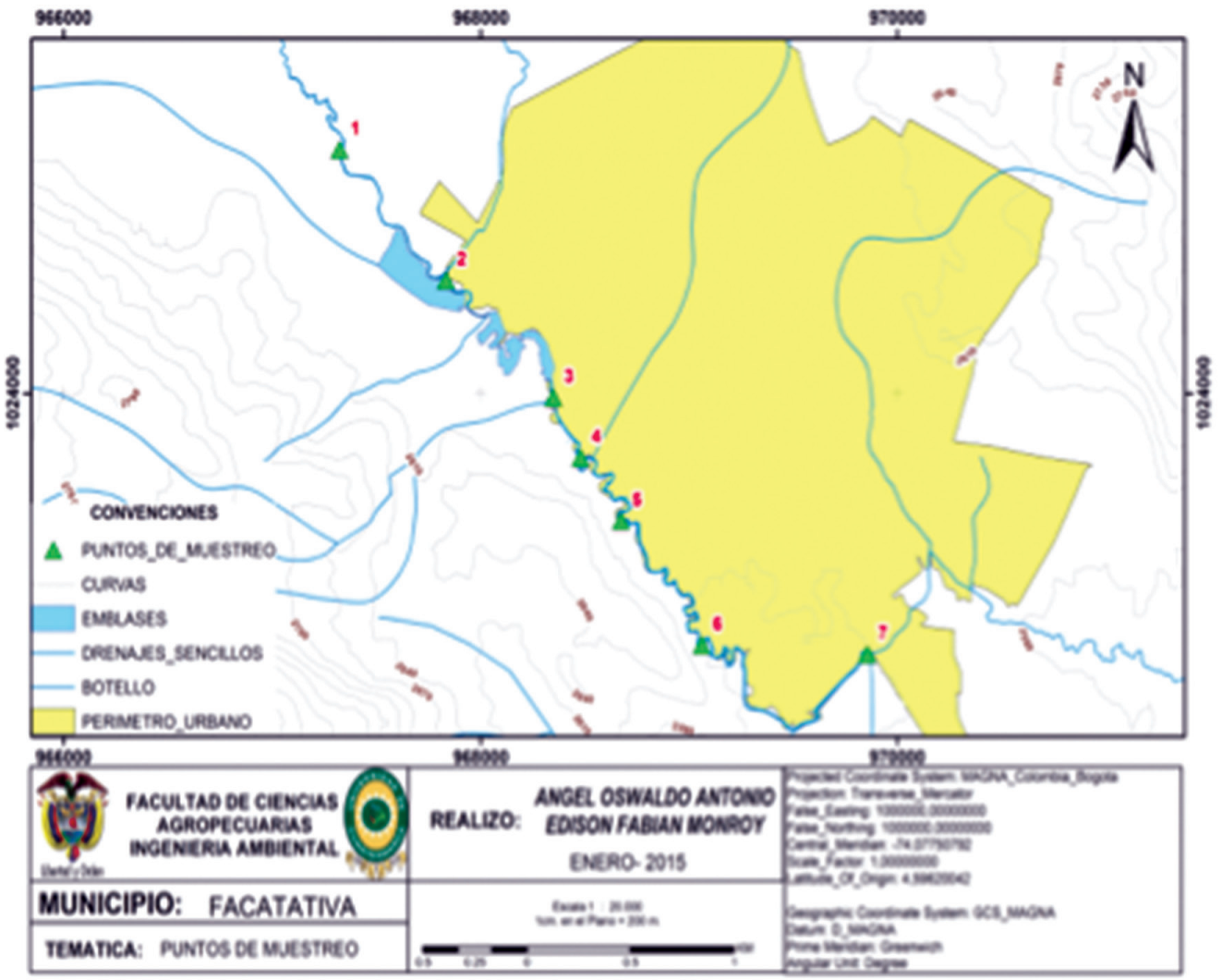

Figura 2. puntos de toma de muestras durante los tres muestreos.

\section{Resultados y discusión}

El primer resultado obtenido, fue el perfil o levantamiento topográfico de la cross section data (Figura 3) de las 100 secciones transversales que se levantaron a lo largo del tramo de estudio. En la Figura 4, se observa un perfil, ejemplo, en el cual se definen las variables adicionales.
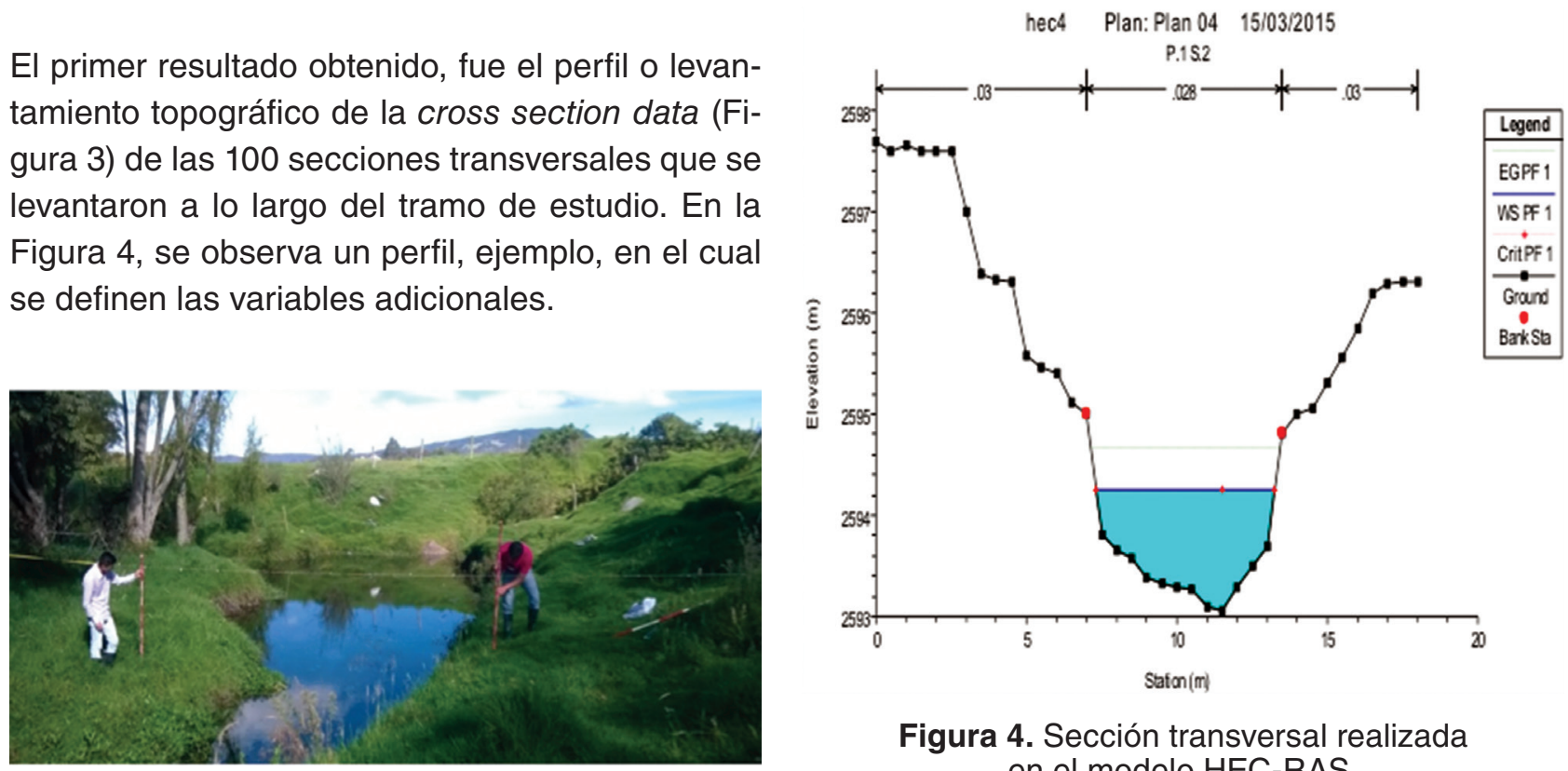

Figura 3. Levantamiento topográfico de las cross section mediante altimetría.

Figura 4. Sección transversal realizada en el modelo HEC-RAS. 
De acuerdo con las secciones transversales y la Geometric Data, se dio inicio a la definición de condiciones de contorno (boundary conditions) para los puntos establecidos en el caso aguas arriba (upstream) y aguas abajo (downstream).
En la Figura 5, se observan las condiciones de contorno definidas en la presente investigación para el río Botello y así continuar con el respectivo modelamiento en el flujo inestable (unsteady), en el cual se puede definir más de una condición de contorno.

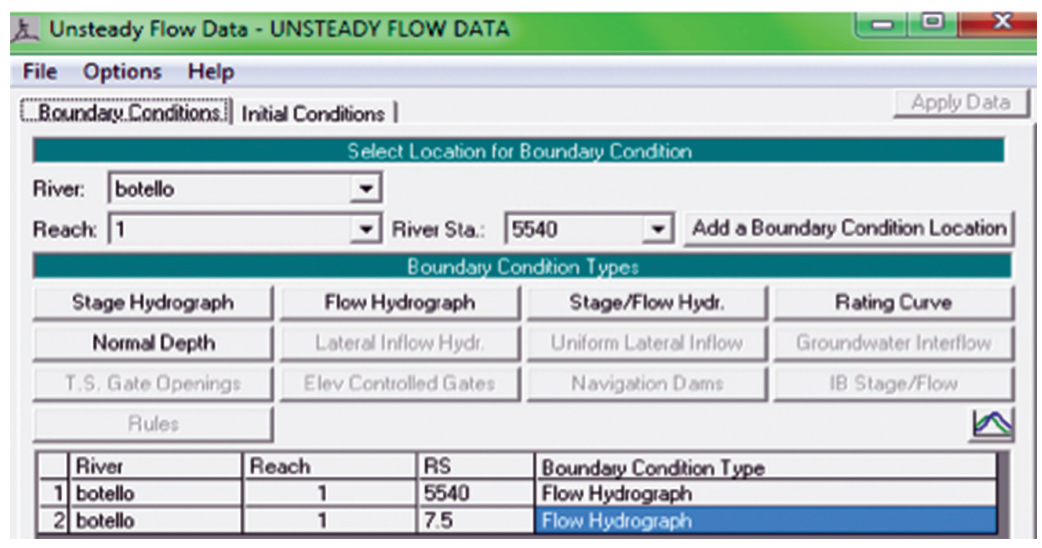

Figura 5. Condiciones de contorno para el río Botello

De este modo, para el Río Botello se definieron dos boundary conditions tipo. Así se estableció el hidrograma de flujo (flow hydrograph) para cada una de las secciones y de esta forma, garantizar posteriormente, una calibración del modelo con los datos obtenidos por el software HEC-RAS (Torres, 2010).

Luego de tener los datos, las condiciones de contorno, y haber culminado los tres pasos metodológicos de la simulación, se llega al paso 4, el cual, consiste en ejecutar la simulación en flujo variable: Run unsteady flow simulation, (Figura 6), donde luego de corregir los errores que se presentaron, se dio inicio al modelamiento hidrodinámico del tramo estudiado, en el cual se obtuvieron los resultados finales de este proceso.

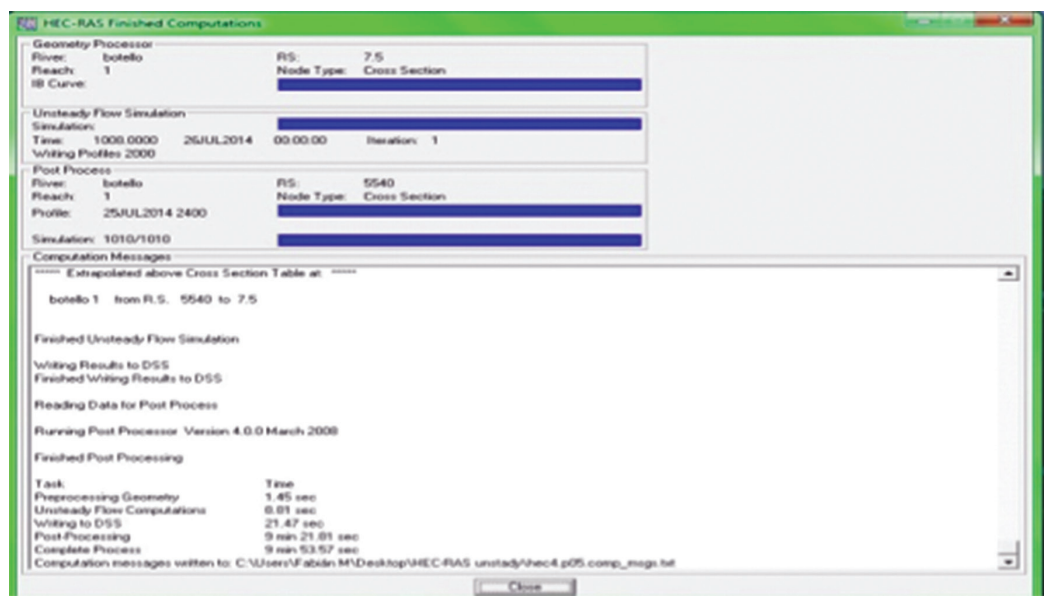

Figura 6. Estabilidad del modelo Hidrodinámico.

La calibración del modelo Hec-Ras se desarrolló mediante el método del error relativo y el error absoluto. Seguidamente se halló el error absoluto de velocidades y se obtuvo un 10.22 $\%$, se utilizaron los datos tomados por los autores como caudales, área del canal, superficie 
de flujo libre, se ejecutó y se calibrò con el área de cada sección y posteriormente, éste arrojó un error absoluto correspondiente al $8.96 \%$; asi mismo sucedió con la superficie del agua (top width), profundidades (depths), y velocidades (velocity). (Garcia \& Chang, 2012).

Finalmente se concluye que con las tres variables que fueron tomadas en campo al momento de calibrar, el modelo arrojó un error absoluto final del 8.06\%, (Figura 7). De acuerdo a pronósticos nacionales, se considera que un pronóstico es acertado siempre y cuando el error no supere el $10 \%$, según Rivera el modelo permite un error entre el 3 y el 33\% según el mismo ARMY.

\begin{tabular}{|c|c|c|c|c|c|c|c|c|c|c|c|}
\hline \multicolumn{12}{|c|}{ 罪Profile Output Table - Standard Table 2} \\
\hline \multirow{2}{*}{\multicolumn{12}{|c|}{ File Options Std. Tables Locations Help }} \\
\hline & & & & & & & & & & & \\
\hline \multirow[t]{2}{*}{ Reach } & \multirow[t]{2}{*}{ River Sta } & \multirow[t]{2}{*}{ Profle } & \multirow{2}{*}{$\frac{\text { E.G. Elev }}{\text { (m) }}$} & \multirow{2}{*}{$\frac{\text { W.S. Elev }}{(\mathrm{m}]}$} & \multirow{2}{*}{\begin{tabular}{|c|} 
Vel Head \\
(m) \\
\end{tabular}} & \multirow{2}{*}{\begin{tabular}{|c|} 
Fich Loss \\
(m) \\
\end{tabular}} & \multirow{2}{*}{$\frac{\text { C\&E Loses }}{\mid \mathrm{m}]}$} & \multirow{2}{*}{ OLeft } & \multirow{2}{*}{\begin{tabular}{|c|} 
Q Charnel \\
$(\mathrm{n} 3 / \mathrm{s})$ \\
\end{tabular}} & \multirow{2}{*}{\begin{tabular}{|l|} 
Q Right \\
(m3/s] \\
\end{tabular}} & Toowith \\
\hline & & & & & & & & & & & $(\mathrm{m})$ \\
\hline 1 & 5540 & $\overline{\text { PF1 }}$ & 2593.55 & 2593.45 & 0.10 & 0.00 & 0.00 & & 1.00 & & 3.56 \\
\hline 1 & 5534 & PF1 & 2593.25 & 2593.15 & 0.10 & 0.10 & 0.00 & & 1.00 & & 3.57 \\
\hline 1 & 4659 & PF1 & 2592.20 & 259207 & 0.12 & 0,00 & 0.00 & & 1.00 & & 265 \\
\hline 1 & 4654 & PF1 & 2592.00 & 2591.87 & 0.12 & 0.11 & 0.00 & & 1.00 & & 2.65 \\
\hline 1 & 3561 & PF1 & 2591.78 & 2591.62 & 0.16 & 0,00 & 0.00 & & 1.00 & 0.00 & 1.92 \\
\hline 1 & 3556 & PF1 & 2591.48 & 2591.32 & 0.16 & 0003 & 0.04 & & 1.00 & 0,00 & 1.92 \\
\hline 1 & 3386 & PF1 & 2591.11 & 2591.09 & 0.02 & 0.01 & 0.00 & 0.00 & 1.00 & 0.00 & 3.96 \\
\hline 1 & 3391 & PF 1 & 2591.10 & 2591.10 & 0.01 & 0.18 & 0.00 & 0.01 & 097 & 0.02 & 4.91 \\
\hline 1 & 3215 & PF1 & 2590.92 & 259091 & 0.02 & 001 & 0,0 & 0.08 & $0 \approx 8$ & 0.04 & 4.49 \\
\hline 1 & 3210 & PF1 & 2590.91 & 259091 & 0.01 & 1.46 & 0.01 & 0.11 & 085 & 0.05 & 4.83 \\
\hline 1 & 2542 & PF1 & 2589.45 & 25893 & 0.09 & 0.07 & 000 & & 1.00 & & 2.18 \\
\hline 1 & 2537 & PF1 & 2589.36 & 259933 & 0.02 & 204 & 0.01 & 0.00 & 099 & 0.01 & 3.81 \\
\hline 1 & 1962 & PF1 & 2587.31 & 2587.30 & 0.00 & 000 & 0.00 & 0.01 & $a s 8$ & 0.01 & 6.59 \\
\hline 1 & 1957 & PF1 & 2587.30 & 2587.30 & 0.00 & 089 & 001 & 000 & 095 & 0.02 & 6.91 \\
\hline 1 & 1722 & PF1 & 2585.40 & 259630 & 0.09 & 001 & 0003 & & 1.00 & & 3.99 \\
\hline 1 & 1717 & PF1 & 2586.33 & 259632 & 0.01 & 1.90 & 0.00 & & 1.00 & & 5.30 \\
\hline 1 & 12.5 & Pf1 & 2584.43 & 2584.42 & 0.01 & 0.21 & 001 & 000 & 098 & 0.02 & 7.10 \\
\hline 1 & 7.5 & PF1 & 2584.21 & 2584.14 & 0.08 & & & & 1.00 & & 5.36 \\
\hline
\end{tabular}

Figura 7. Calibración del modelo hidrodinámico.

Luego, se procede a observar los resultados obtenidos en la modelación hidrodinámica, lo cual permitió definir que los lugares con mayor susceptibilidad a inundaciones en temporada de lluvias son: el punto 2, 8 y 9 que corresponden a 3 de los 9 puntos monitoreados.

El río Botello, se caracteriza por presentar meandros a lo largo de su recorrido, característico de los cauces que se encuentran en zonas de baja pendiente, lo cual influyó, en la investigación a la hora de definir las zonas de inundación, debido a que las zonas meándricas generan disminución de la velocidad y represamientos de la corriente en temporadas bajas.

\section{Modelo matemático planteado para el estudio mediante el algoritmo empleado por Hec-Ras 4.0.}

Para el flujo inestable, HEC-RAS resuelve el plano, dinámico, 1-D- la ecuación de Saint
Venant utilizando un método de diferencia implícita, finito. La inestabilidad de resolución de ecuaciones de flujo es una adaptación del paquete UNET del Dr. Robert L. Barkau. (Renata, 2014).

La solución de las ecuaciones de Saint-Venant en 1D ha sido implementada en varias aplicaciones de software comunes usualmente a través de métodos numéricos y en particular por diferencias finitas, entre los más reconocidos se pueden mencionar los siguientes: Hec-Ras, Mike 11, EPASWMM, Storm And Sanitary Analysis.

Para obtener las ecuaciones de Saint-Venant se puede deducir directamente de las mismas, utilizando las leyes de la conservación de la masa y de la cantidad de movimiento, las cuales se pueden aplicar a los cauces de sección arbitraria, incluso no prismáticos. 
Las ecuaciones de Saint-Venant para canales no prismáticos que resultan son mostradas en las Ecuaciones 1 y 2 :

$$
\begin{aligned}
& \text { 1. } \frac{\partial}{\partial t} U+\frac{\partial}{\partial x} F=H \\
& \text { 2. } U=\left(\frac{A}{Q}\right) ; F=\left(\frac{Q}{\frac{Q^{2}}{A}+g I_{1}}\right) ; H=\left(\frac{0}{g I_{2}+g A\left(S_{0}-S_{f}\right)}\right)
\end{aligned}
$$

La solución numérica está dada por la Ecuación 3: Continuidad en el canal principal

$$
\text { 3. } \frac{\partial I_{1}}{\partial s}=I_{2}+A \frac{\partial h}{\partial x}
$$

Hec-Ras no implementa directamente las ecuaciones de Saint-Venant en su forma tradicional, sino las adapta, primero a la geometría típica del cauce, que suele consistir en un canal principal por donde discurre el caudal aguas abajo, y en una llanura de inundación, formada por una avenida de agua.

Teniendo en cuenta que no toda el área mojada de la sección transversal de un río transporta agua, se descompone el área total de la sección Ar en una Zona con transporte efectivo A, y una zona de almacenamiento (S), o área de la sección donde no hay transporte y remplazando $(\mathrm{Ar}=\mathrm{A}+$ S) en la ecuación se obtiene la Ecuación 4

$$
\text { 4. } \frac{\partial A}{\partial t}+\frac{\partial s}{\partial t}+\frac{\partial Q}{\partial x}=q 1
$$

Para abordar el problema del canal principal y la llanura de inundación, los programadores del HEC-RAS han considerado de manera separada el movimiento de agua en estas dos regiones. Según (Huaman, 2013) de esta forma se obtuvieron dos Ecuaciones 5 y 6 :

$$
\text { 5. } \frac{\partial Q_{C}}{\partial x_{c}}+\frac{\partial A}{\partial t}=q_{f}
$$

Continuidad en la llanura de inundación

6. $\frac{\partial Q_{f}}{\partial x_{f}}+\frac{\partial A_{f}}{\partial t}+\frac{\partial S}{\partial t}=q_{c}+q_{f}$

Estas ecuaciones son las que emplea el software HEC-RAS para realizar el modelamiento en el caso de estudio. Luego de estar calibrado el modelo y conocer las ecuaciones determinísticas, se procede a mostrar una serie de escenarios en las que se evidencian los niveles críticos de caudal (temporada seca) y cambios abruptos en los niveles de caudal (temporada lluviosa.) como se observa en la Figura 8.

La temporada seca que se evaluó, corresponde a los datos tomados por los autores del año 2014; mientras que para los otros escenarios se tomaron caudales históricos de eventualidades desde hace años, en estos se realizó la misma ruta metodológica. En el año 2006 se levantó el jarillón lo cual hace que estos riesgos disminuyan, y consecuentemente la investigación por la parte del HecRas encontró que durante la temporada lluviosa este cauce superaba los over bank station en los puntos en que el río disminuía su ancho. 


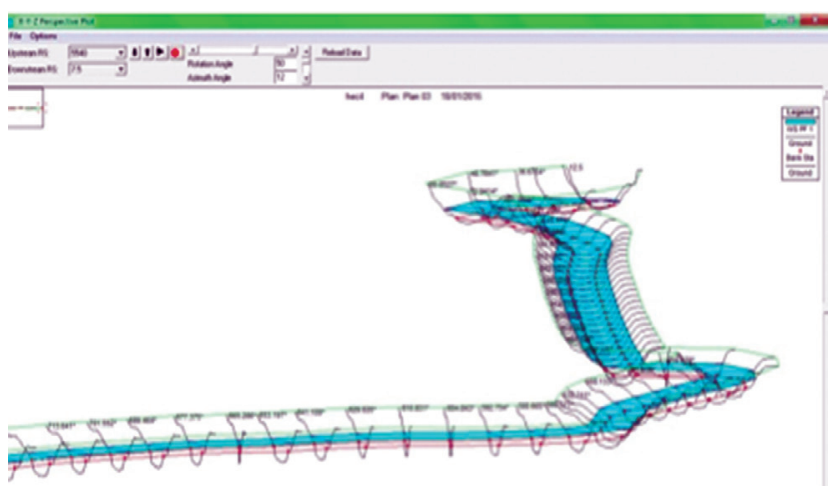

Figura 8. Excesos de caudales y superación de Over bank.

\section{Temporada seca.}

A continuación, se presentan los resultados correspondientes a la temporada seca, y como se observa en las Figuras 9, 10 y 11, se nota que el caudal del que disponía el cauce, era un caudal de riesgo bajo para las inundaciones, donde de acuerdo con esto y la baja velocidad de flujo, se alteran algunas variables de la calidad del agua.

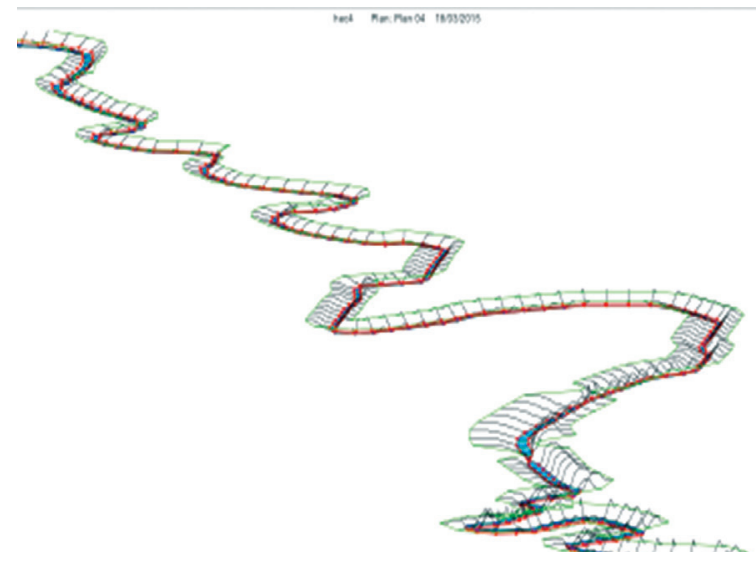

Figura 9. Modelamiento temporada seca tramo punto 3-5.

Además en la Figura 10, se observa el perfil del cauce donde se puede apreciar mejor el comportamiento del espejo de agua y la variación de altura la cual es muy baja para el recorrido del mismo con pendiente menor del $3 \%$.

En las figuras anteriores, se analiza igualmente, que el caudal de estudio inicial es un caudal de baja intensidad, debido a que no supera el $50 \%$ de la altura para alcanzar la cota de inundación (over bank). En la Figura 11, se observa mejor el flujo hídrico donde se evidencia que a lo largo de unos pocos perfiles, el flujo se encuentra en su cauce natural (channel).

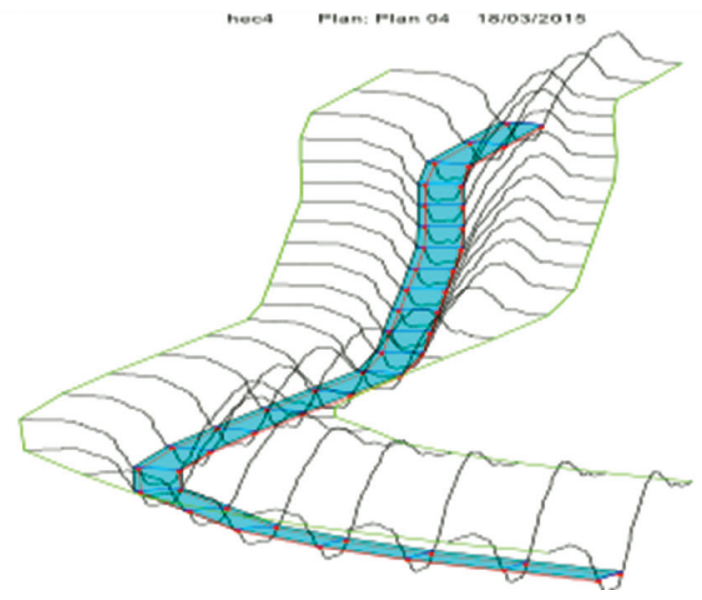

Figura 10. Perfiles del cauce y su flujo hídrico.

\section{Temporada transitoria (seca-Iluviosa).}

En el período transitorio, se alcanzó un caudal de $1.6 \mathrm{~m}^{3} / \mathrm{seg}$, donde validando estos caudales monitoreados en el software y calibrarlo con su respectivo procedimiento, se observan claramente las zonas vulnerables a inundaciones (Figuras 12 y 13). En ésta sección se pudo observar tanto en la simulación como en tiempo real que esta zona, efectivamente se encontraba inundada en esta escala de tiempo y espacio como se tiene evidencia en la Figura 13. Situación que permite corroborar la fiabilidad del modelo.

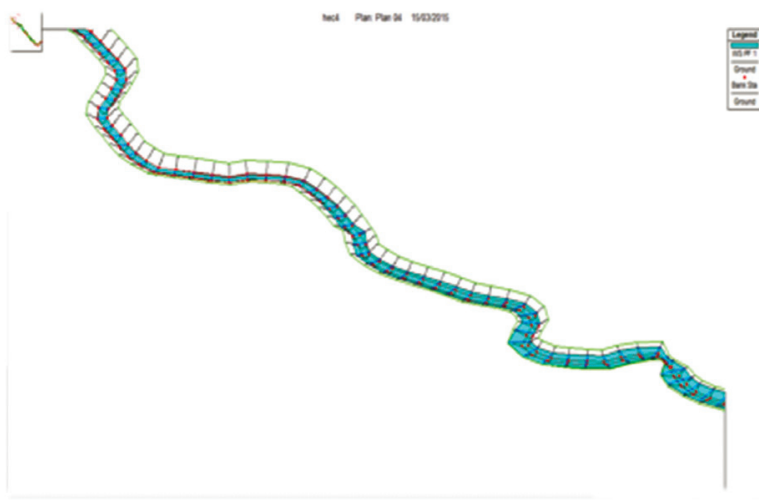

Figura 11. Tramo del río Botello comprendido entre los puntos 8 y 9. 
En la Figura 12 se observa, que la inundación que se presenta tiene el siguiente comportamiento: hacia el lado izquierdo, alcanza un límite cercano a la proximidad del desbordamiento sobre la zona urbana y hacia el lado derecho correspondiente con la zona rural, se desborda, debido a que la cota es más baja, afectando cultivos y actividades agropecuarias que se desarrollan en el área al momento de la inundación. Alterando así, los procesos agrícolas que se encuentran en la rivera, además de ser el recurso hídrico quien sufre los impactos en la alteración de la composición química y física del agua en términos de calidad.

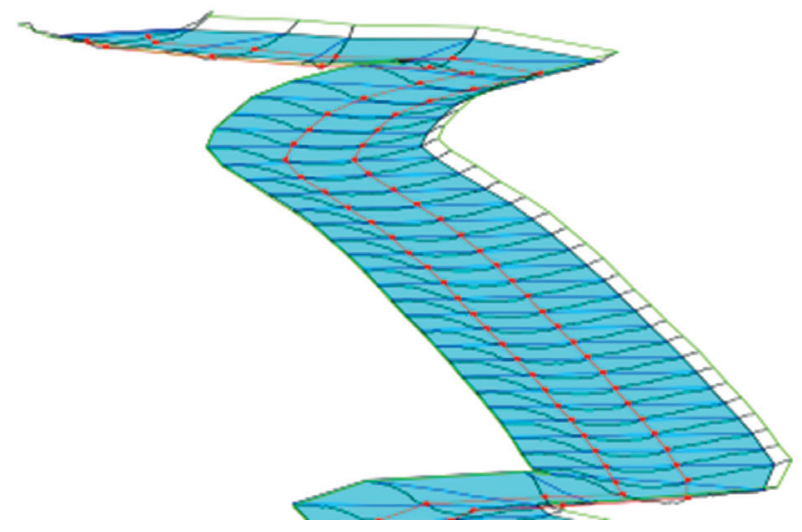

Figura 12. Lugar de la inundación presentada en la temporada transitoria en la sección 9.

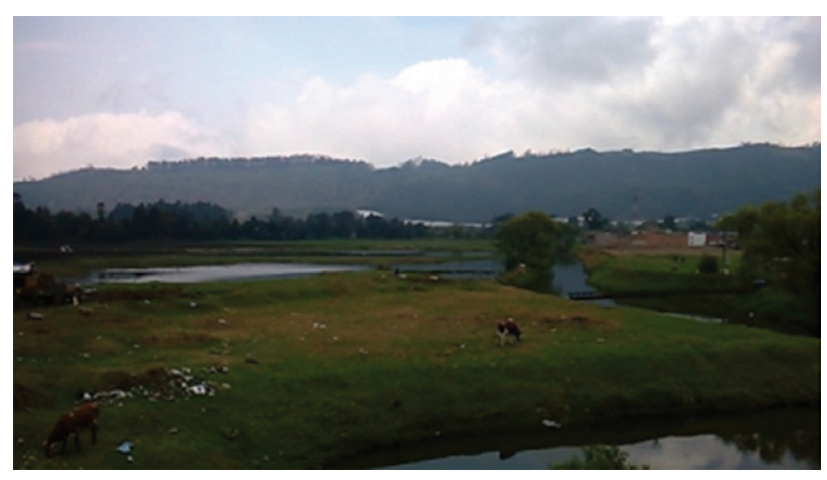

Figura 13. Inundación en el punto 9 para el mes de Octubre 2013.

Otro de los ejemplos, que permite corroborar la fiabilidad del modelo, se evidencia en esta temporada en el punto 1 lugar de inicio de monitoreo.

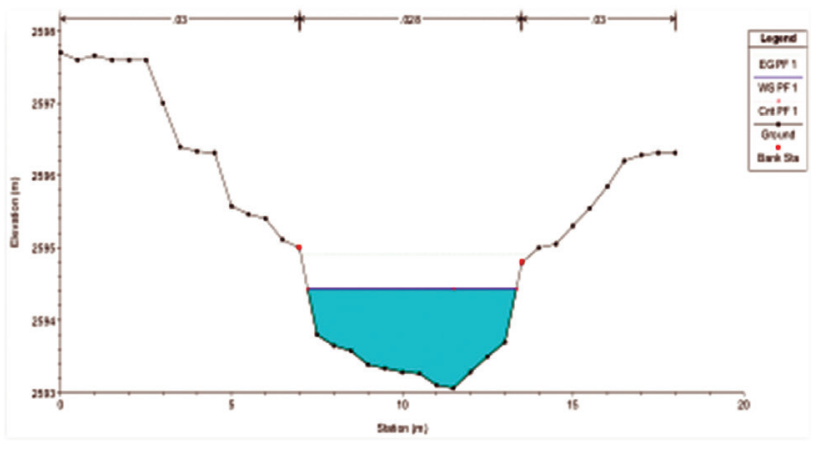

Figura 14. Perfil 1 inicio del muestreo temporada transitoria.

En la Figura 14, se evidencia que el caudal medido para este día no supera la cota de inundación (over banks) y las profundidades obtenidas en campo son muy aproximadas a las obtenidas en el modelo con un error menor al 3\%. En la Figura 15 , se observa otra de las evidencias, que muestran que estos resultados sin lugar a duda son similares a la realidad que se vive alrededor del río Botello.

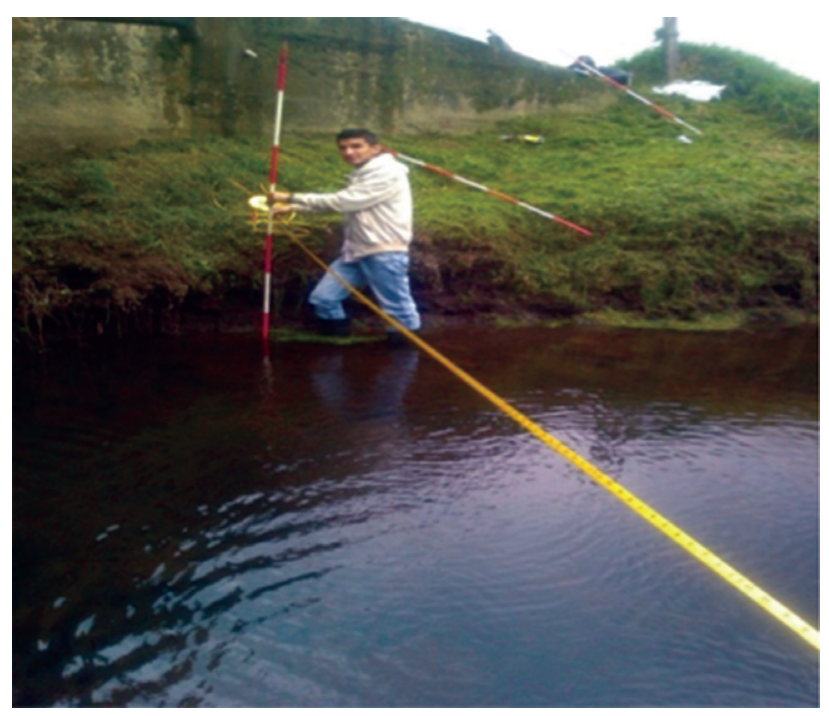

Figura 15. Muestreo punto 1.

Finalmente, se analizaron a modo de resumen los resultados más importantes para las dos temporadas con el fin de corroborar la veracidad del modelo. 
Dentro de los resultados obtenidos en la investigación, se destaca el gran aporte de información de este tipo para el municipio de Facatativá, debido a que no existía ninguna información de batimetría y modelación matemática del río Botello en la zona urbana del municipio, ni estudios de calidad del agua en el tramo de estudio, únicamente en la empresa Aguas de Facatativá.

\section{Resultados de la calidad del agua}

A continuación, se muestran los emitidos por la Empresa Aguas de Facatativá para las 20 pruebas realizadas en las (3) etapas de monitoreo. En estas, se evalúan las variables de $\mathrm{pH}$, temperatura, turbiedad, color aparente, conductividad, DQO, DBO5, fosfatos, hierro, nitratos, nitritos, manganeso, oxígeno disuelto, solidos disueltos totales, además, de evaluar dos parámetros microbiológicos importantes: E- coli y Coliformes totales.

Se analizan los parámetros establecidos en el presente estudio. En las siguientes ilustraciones se asocian los colores azul, verde y naranja, para facilitar el análisis de resultados, los cuales indican:

Azul: Primera etapa de muestreo.

Verde: Segunda etapa de muestreo.

Naranja: Tercera etapa de muestreo

\section{Potencial de Hidrógeno: pH}

Es una de las más frecuentes e importantes pruebas usadas en monitoreos de aguas. Se utiliza para conocer medidas de alcalinidad, dióxido de carbono, y muchos otros equilibrios acido-base (Who, 1996), haciendo referencia a la concentracion y actividad del ion hidrógeno. El pH de la mayoría de las aguas naturales está estandarizado entre 6.0 y 8.5 . Pueden ocurrir valores más bajos en aguas diluidas, altas en contenido de materia orgánica, y valores más altos en aguas eutróficas (American Public Health Association, 2012).

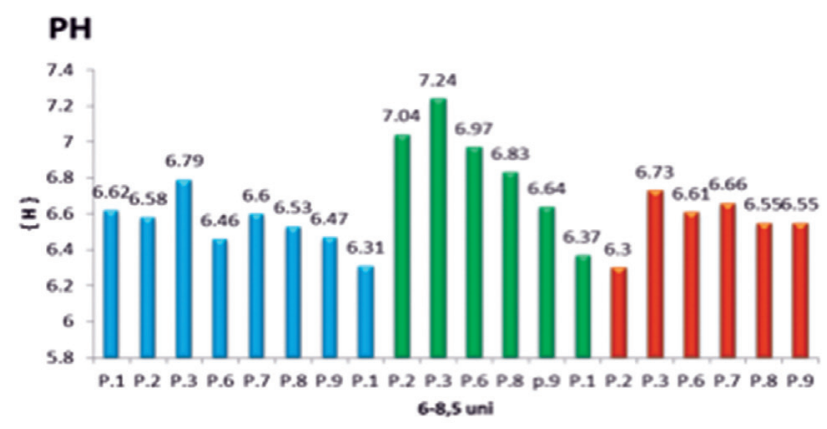

Figura 16. $\mathrm{PH}$ del río Botello en la zona urbana de Facatativá.

Como se evidencia en la Figura 16, el pH medido a lo largo de los 9 puntos de muestreo, en definitiva, se encuentra dentro los estándares permitidos por la Organización Mundial de la Salud (Who, 1996), con respecto a calidad de agua superficial en las tres etapas. Es de resaltar, que los valores más altos se observaron en la segunda etapa, que se realizó en una temporada seca, debido a que es afectada por los bajos caudales y escaso movimiento de las aguas, siendo el $\mathrm{pH}$ influenciado por el rápido crecimiento y respiración de plantas, como lo es el caso propio de la elodea (Elodea nuttallii).

\section{Turbiedad.}

La WHO, define que la turbidez del agua superficial no debe superar en ningún caso las 5 NTU, y estará idealmente por debajo de 1 NTU [4]. De acuerdo con la Figura 17, y los datos obtenidos en el primer muestreo realizado en el río BoteIlo, la turbiedad en todos los puntos se encuentra en los estándares permitidos. Mientras que, en el segundo muestreo se encuentra que en el punto 1, 3, 6, 8 y 9 la turbiedad alcanza valores máximos de $78.1 \mathrm{NTU}$, producto de la temporada seca evaluada. 


\section{TURBIEDAD}

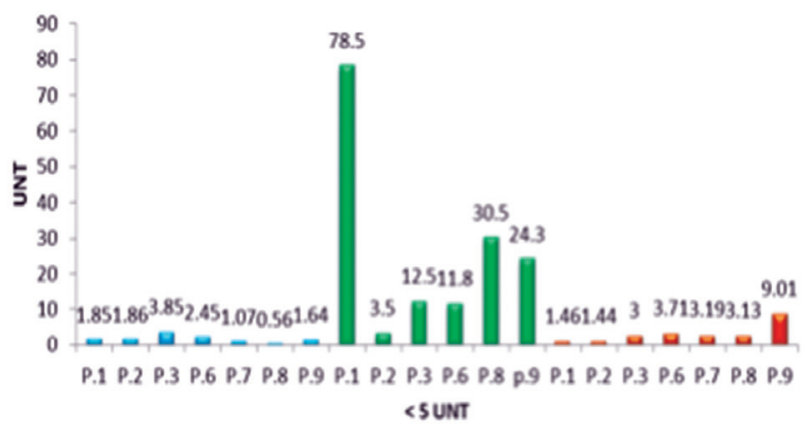

Figura 17. Turbiedad del río Botello en la zona urbana de Facatativá

Finalmente, se encontró que solo en el punto 9 de muestreo, existen valores que sobrepasan los estándares establecidos por la WHO, siendo la causa de estos los vertimientos que se sitúan cerca a este tramo del río. Por tal motivo, en términos finales, el agua superficial del río Botello es más confiable en temporada lluviosa y transitoria (seca - lluviosa) que en temporada seca.

\section{Demanda Química de Oxígeno (DQO).}

La demanda química de oxígeno (DQO), es una medida del equivalente de oxígeno de la materia orgánica en una muestra de agua que es susceptible a la oxidación por una potente sustancia química oxidante, tal como el bicromato potásico (Garcia \& Chang, 2012)

\section{DQO}

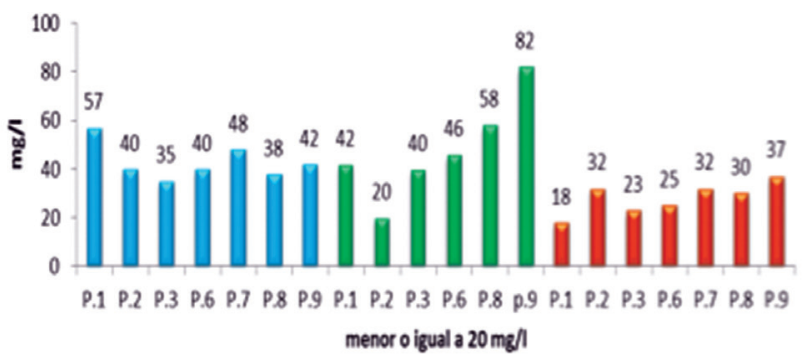

Figura 18. DQO del río Botello Zona urbana de Facatativá

Con referencia a los valores obtenidos en las muestras representados en la Figura 18, es agua que durante la primera fase y la segunda sobrepasan los valores permitidos de $20 \mathrm{mg} / \mathrm{l}$. Mientras que en la tercera fase el punto 1 se encuentra dentro de los valores permitidos. Siguiendo este monitoreo se encuentra un crecimiento de esta variable alcanzando valores de $37 \mathrm{mg} / \mathrm{l}$ siendo estos valores más bajos que los de las fases anteriores. Concluyendo esta variable se permitió establecer que no se encuentra dentro de los picos establecidos por la WHO.

\section{Fosfatos.}

La fuente principal de los fosfatos orgánicos son los procesos biológicos. Estos pueden generarse a partir de los ortofosfatos en procesos de tratamiento biológico o por los organismos acuáticos del cuerpo hídrico. Concentraciones relativamente bajas de complejos fosforados afectan el proceso de coagulación durante el tratamiento del agua (Who, 1996).

\section{FOSFATOS}

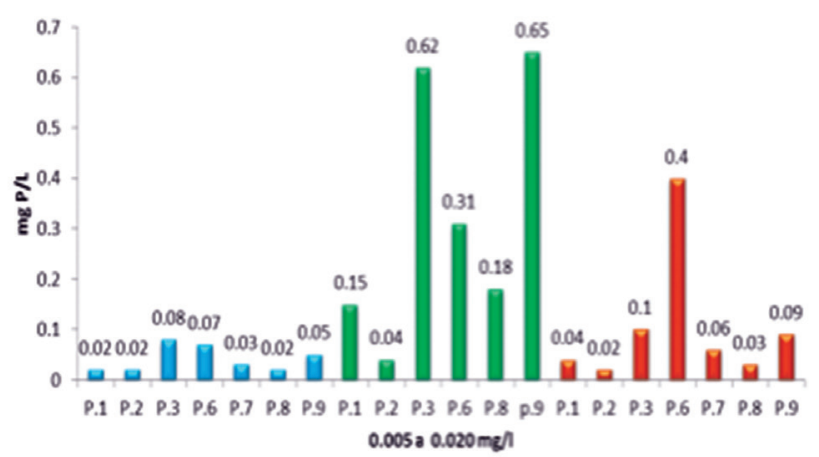

Figura 19. Fosfatos en el río Botello zona urbana de Facatativá.

Por otro lado los fosfatos que se encuentran presentes en el río Botello varían de 0.02 a 0.65 mg/l, donde se evidencia que durante la primera fase los fosfatos presentan niveles que están en los valores máximos permitidos por la WHO, mientras que en la segunda fase se encuentran valores más altos debido a que la velocidad de corriente es mínima y se dan procesos en aguas estancadas aumentando el crecimiento de las plantas acuáticas; finalmente en la tercera fase los valores obtenidos fueron muy variables presentando 
el valor más alto, según la Figura 19 para el punto 6 la gran cantidad de plantas acuáticas aumentan los fosfatos. Por tal motivo el agua no cumple los valores en ningún punto muestreado y se evidencia un exceso de fosforo en los límites permitidos lo cual indica la presencia de contaminación y son en gran parte responsable de condiciones eutróficas y gran crecimiento de plantas acuáticas a lo largo del sistema de estudio.

\section{Oxigeno Disuelto.}

La cantidad de oxígeno que está en el agua se denomina oxígeno OD disuelto. La solubilidad es directamente proporcional a la presión parcial. Como lo establece la WHO el Oxígeno disuelto (OD) en las aguas frescas en el nivel del mar oscila entre $15 \mathrm{mg} / \mathrm{l} \mathrm{a} 0^{\circ} \mathrm{C}$ y $8 \mathrm{mg} / \mathrm{l}$ a $25^{\circ} \mathrm{C}$. Las concentraciones en aguas no contaminadas son por lo general menor a $10 \mathrm{mg} / \mathrm{l}$. En el agua potable por lo general puede ser detectado por los consumidores como resultado de un mal olor y sabor (Who, 1996).

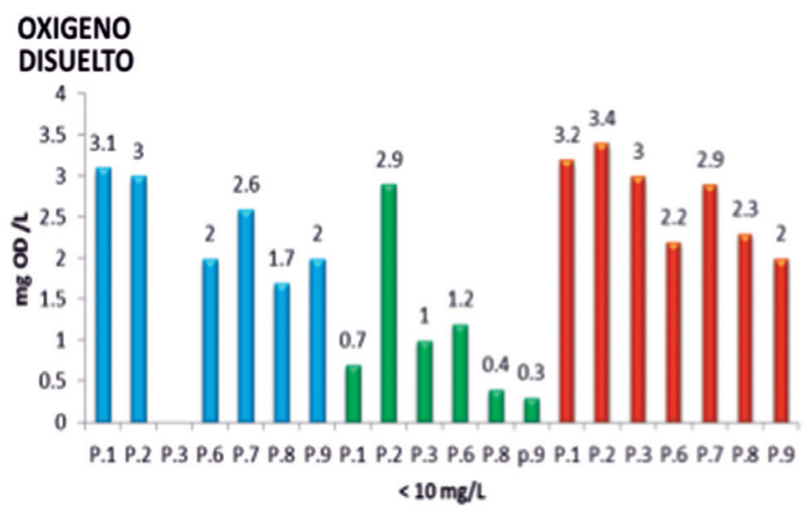

Figura 20. OD en el río Botello zona urbana de Facatativá

En relación con lo establecido el agua del Río Botello debe presentar OD mayor o igual a $10 \mathrm{mg} / \mathrm{L}$ para fuentes superficiales. Analizando los valores presentados en la Figura 20 se establece que hay un déficit de oxígeno disuelto lo que causa cambios físicos en el color y sabor, además estos valores bajos se deben a que no hay saltos hidráulicos los cuales ayudarían a aumentar estos valores.

\section{Nitratos.}

El ion nitrato $\left(\mathrm{NO}_{3}^{-}\right)$Es la forma común de nitrógeno combinado se encuentra en aguas naturales. Puede ser bioquímicamente reducido a nitrito $\left(\mathrm{NO}_{2}{ }^{-}\right)$por los procesos de desnitrificacion, por lo general en condiciones anaeróbicas. El ion nitrato se oxida rápidamente a nitrito. Las fuentes naturales de nitrato en las aguas superficiales incluyen rocas ígneas, drenaje de tierras de plantas, animales y escombros.

Las concentraciones superiores a $5 \mathrm{mg} / \mathrm{l} \mathrm{NO}_{3}$ generalmente indican la contaminación por desechos humanos o animales o escurrimiento de fertilizantes. En los casos de contaminación extrema, las concentraciones pueden llegar a 200 mg/l.

\section{NITRATOS}

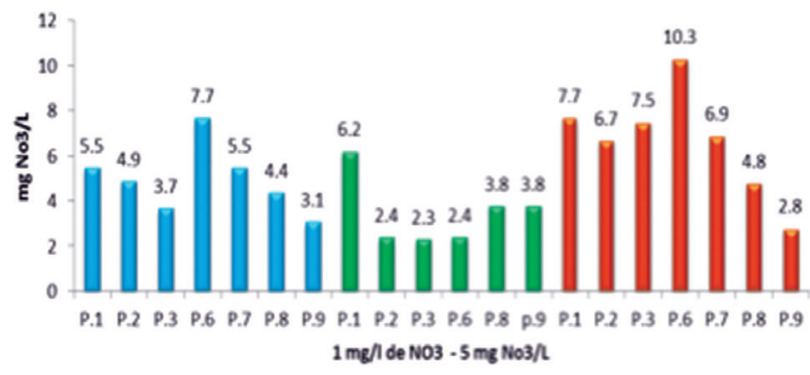

Figura 21. Nitratos en el río Botello en la zona urbana de Facatativá

La Organización Mundial de la Salud (WHO) recomienda límite máximo de $\mathrm{NO}_{3}$, hasta $5 \mathrm{mg} / \mathrm{l}$ de $\mathrm{NO}_{3}$, pero a menudo menos de $1 \mathrm{mg} / \mathrm{l}$ de $\mathrm{NO}_{3}$. Las concentraciones superiores a $5 \mathrm{mg} / \mathrm{l}$ por lo general indican la contaminación por desechos humanos o animales, o escorrentía de fertilizantes. En los casos de contaminación extrema, las concentraciones pueden llegar a 200 mg/l NO 3 (Departament of health and human services, 2009).

De acuerdo a estas recomendaciones y los resultados mostrados anteriormente en la Figura 21 se encuentra que durante la primera y segunda fase, en el punto 1 supera el valor permitido, esto debido que en la parte rural es mayor el uso de los 
abonos inorganicos para la agricultura y fertilizacion de forrajes en el caso de la ganaderia. En último lugar en la fase tres los valores de nitratos se comportan de una manera muy diferente, esto debido a lo siguiente en el punto 1 el uso de fertilizantes es mas bajo hasta el punto 5. Apartir de este y hasta el 9, se encuentra un valor muy alto lo cual corrobora que efectivamente hay cultivos aledaños al punto.

\section{Escherichia coli (E. Coli)}

La Escherichia coli pertenece a un grupo de bacterias presentes en el intestino del ser humano y animales, siendo la gran mayoría inocuas en ellos. Los rumiantes, y en particular el ganado bovino y ovino, son el principal reservorio de estas bacterias. Los animales portadores no muestran ningún signo clínico y eliminan las bacterias E.coli por las heces (Ojeda, 2012)

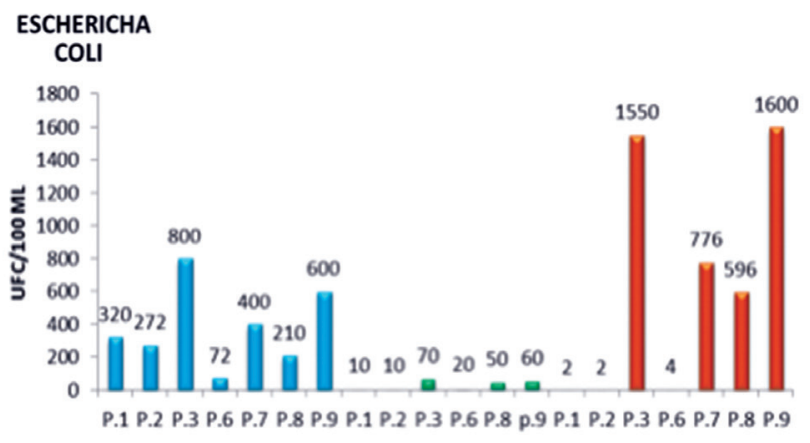

Figura 22. E. Coli. En el río Botello Zona urbana de Facatativá.

Con las características mencionadas sobre la E.coli. y los resultados obtenidos en los tres monitores que se evidencian en la Figura 22 se analiza que durante la primera etapa estos valores son un poco elevados debido a que los vertimientos se mezclan con la corriente hídrica, disolviendo estas concentraciones y asi mismo descendiendo estos valores. Para la segunda fase estos niveles son bajos ya que disminuye la escorrentía y los vertimientos no alcanzan a llegar al punto de muestreo debido al bajo flujo hidráulico. En la fase final los valores son muy elevados debido a las condiciones meteorológicas del día anterior al muestreo se presentaron precipitaciones en la zona, lo cual hace que la escorrentía de las zonas de mayor pendiente vaya a la de menor pendiente (cauce principal), llevando consigo materia portadora de $E$. coli, disuelta en ella.

\section{Conclusiones}

Entorno a las variables hidrodinámicas del Río Botello monitoreadas se encontró que la variación y aumento de los caudales afecta principalmente al punto 9 donde cada vez que se presentan precipitaciones intensas en un corto tiempo se inunda la misma zona.

La modelación del río Botello se constituyó como una herramienta indispensable para la gestión integral del recurso hídrico en el municipio y debido a esto, se pueden derivar nuevos proyectos que tiendan a mitigar los impactos ambientales y económicos que se presentan en la zona.

Las zonas más susceptibles a inundación se evidenciaron en el punto $9 \mathrm{y}$ en un periodo de retorno estudiando los datos meteorológicos históricos, se encuentra que la inundación que se presentó en el año 2006 fue desde el punto 3 hasta el punto 9, medida que obligo a la administración municipal a crear el jarillón cuya construcción disminuyó en gran parte las inundaciones

La influencia antrópica en torno al río Botello acarrea grandes problemas de calidad a la oferta del recurso hídrico para los habitantes del municipio que suplen sus necesidades básicas.

La disminución de caudales afecta principalmente a la empresa Aguas de Facatativá la cual provee agua para el municipio en una cobertura del $100 \%$ en el sector urbano, lo cual causa una sobre explotación del recurso hídrico en la zona urbana de Facatativá.

El modelo unidimensional Hec-Ras posee todas las capacidades necesarias para realizar una simulación en las condiciones previstas en el estudio. 
Luego de dar a conocer estar investigación a la CAR, ellos realizaron una adecuación hidráulica del Río, reforzando jarillones y removiendo sedimentos, los cuales debido a los altos contenidos de hierro, magnesio y otras sales disminuye la eutrofización en el río.

La modelación del río Botello se constituyó como una herramienta indispensable para la gestión integral del recurso hídrico en el municipio.

Se encontró que las zonas más susceptibles a inundación son en el punto 7 y 8 , siendo la de mayor magnitud en el punto 9, donde siempre ocurren debido a que la pendiente del cauce es muy baja y el jarillòn se encontraba alterado.

\section{Agradecimientos}

Los autores expresan un agradecimiento a Dios, a la Universidad de Cundinamarca, al grupo de investigación Axioma, a la empresa aguas de Facatativá, a nuestro director Diego Bedoya, a las profesoras Jaddy Castañeda y Angélica Bravo, a nuestras familias y compañeros de carrera.

\section{Literatura citada}

1. American Public Health Association, A. W. (2012). Standard Methods for the examination of water and wastewater. 22th ed. Cap. 1060 collection and preservation of sam.

2. Blade, E. (2009). Modelación Numérica en río s en régimen permanente y variable. Una visión A partir del Modelo HEC-RAS. 1 ra edición.

3. Departament of Health and Human Services. (2009). Las bacterias. Recuperado de http://epi.publichealth. nc.gov/oee/docs/Las_Bacterias_Coliformes_WellWaterFactSt.pdf

4. Facatativá., A. M. (2001). Plan de Ordenamiento Territorial. Facatativa.

5. Garcia, F. \& Chang, G. y. (2012). Calibration and validation 3D in the Santa Marta coastal area (Colombia). Recuperado de http://www.scielo.org.co/scielo. php?script=sci_artte
6. Huaman, B. A. (2013). Tesis presentado por el Bachiller en Ingeniería Mecánica de Fluidos, Universidad nacional mayor de san marco, Modelamiento numérico de una onda de avenida generada por la rotura de una presa de tierra y su estimación de riesgo- aplicación a la presa Yuracmay, Lima.

7. Icontec. (1996). Norma Técnica Colombiana 813, 56676. Normas oficiales para la calidad del agua y su respectivo muestreo en Colombia. Bogota.: Instituto Colombiano de Normas Técnicas y Certificación.

8. Mautaco, G. (2014). Analisis morfometrico de la cuenca y la red de drenaje del río Zadorra y sus afluentes, aplicado a la peligrosidad de inundaciones. (p. y. Departamento de Geografia, Ed.) Universidad del País Vasco.

9. Ministerio de vivienda. (2010). En Cundinamarca Minvivienda ejecuta obras por $\$ 25.573$ millones en el sector de agua y saneamiento básico. Recuperado de: http:// www.minvivienda.gov.co/Paginas/Sala_de_Prensa/ Noticias/2014/En-Cundinamarca-Minvivienda-ejecutaobras-por-\$25-573-millones-en-el-sector-de-agua-ysaneamiento-b\%C3\%A1sico-.aspx

10. Ojeda, C. (2012). Caracterización fisicoquímica y parámetros de calidad del agua de la planta de tratamientos de agua de Barrancabermeja. 52-58. Bucaramanga, (Teiss de pregrado) Ingeniero Químico: Universidad Industrial de Santander.

11. Renata, J. (2014). Stochastic flood forecasting system. Geoplanet.

12. Secretaría de Desarrollo Agropecuario. (2012). Perfil ambiental de Facatativá. Informe General, Facatativa.

13. Torres, Q. E. (2010). Aplicación del modelo de simulación hidráulica del HEC-RAS para la emisión de pronósticos hidrológicos de inundaciones en tiempo real, en la cuenca media del rio Bogotá - sector alicachin. Recuperado de Revista ingenio libre: http:// www.unilibre.edu.co/revistaingeniolibre/revista10/articulos/aplicaci\%C3\%B3n-del-modelo-de-simulacionhidr\%C3\%A1ulica-hec-ras.pdf

14. Universidad del Valle. (2010). Guía básica para la carecterización Morfométrica de cuencas Hidrográficas.

15. Us army corps of engineers. (2010). Hydrologic Engineering Centers River Analysis System (HEC-RAS).

16. Who, U. U. (1996). Water Quality Assessments. A Guide to Use of Biota, Sediments and Water in Environmental Monitoring. Second Edition; Editorial E\&FN Spon, Behalf

\section{Conflicto de Intereses}

Los autores declaran no tener ningún conflicto de intereses 
